\title{
Comparison of the Effects of Bare Metal Stents and Drug Eluting Stents on C- Reactive Protein Levels
}

\author{
Ozyuncu Nil*, Gulec Sadi, Ozcan Ulas Ozgur, Goksuluk Huseyin, Gerede MenekseDemet, Caglar Nail, \\ Erol Cetin
}

Department of Cardiology, Ankara University School of Medicine, Ankara, Turkey

Corresponding author: Ozyuncu Nil, Department of Cardiology, Ankara University School of Medicine, Ankara, Turkey, Tel: 90312 5708991; E-mail: nilozyuncu@yahoo.com

\begin{abstract}
Aim: It's suggested that drug eluting stents (DES) may have systemic anti inflammatory properties and this can play a role in decreased restenosis rates. We aimed to compare bare metal stents (BMS) and DES on their effects on C-reactive protein (CRP) levels, a good marker of systemic inflammation. We also aimed to investigate the relation between the inflammation levels and myonecrosis and adverse cardiac events.

Methods: Patients undergoing elective stent implantation were grouped as BMS (n $=70)$ and DES $(n=42)$. Basal and $24^{\text {th }}$ hour postprocedural CRP and CKMB levels were measured and the difference $(\Delta)$ was compared between the groups. The patients were followed up for adverse cardiac events for one year.

Results: Mean age was $62 \pm 11$ and $75 \%$ were males. There was significant CRP rise in both groups at $24^{\text {th }}$ hour, but the $\Delta$ CRP was $2.1(0.5-6.2) \mathrm{mg} / \mathrm{L}$ in BMS and $2.3(0.2-5.2) \mathrm{mg} / \mathrm{L}$ in DES group, the difference wasn't statistically significant ( $\mathrm{p}$ $=0.703) . \triangle \mathrm{CKMB}$ and adverse cardiac event rates were similar between the two groups $(\mathrm{p}=0.897$ and $\mathrm{p}=0.785)$. There was no correlation between $\triangle \mathrm{CRP}$ and $\triangle \mathrm{CKMB}(\mathrm{r}=-0.090$ and $\mathrm{p}=0.459$ for BMS, $\mathrm{r}=0.158$ and $\mathrm{p}=0.318$ for DES). The effect of $\triangle C R P$ on the incidence of adverse cardiac events was not significant ( $p=$ 0.349 for BMS, $\mathrm{p}=0.135$ for DES).

Conclusion: Our findings reveal that patients with BMS and DES implantation exhibit similar grade of systemic inflammation after the procedure. At similar levels of systemic inflammation, the local anti-inflammatory properties of DES can play a role at decreased restenosis rates.
\end{abstract}

Received Date: September 23, 2015 Accepted Date: December 18, 2015 Published Date: December 27, 2015

Citation: Nil, O, et al. Recent Advances in Management of Anti-Coagulation in Atrial Fibrillation. (2015) J Heart Cardiol 1(3): 1-7.

Keywords: Bare metal stent; Drug eluting stent; CRP; Inflammation; CKMB

DOI: $10.15436 / 2378-6914.15 .015$

\section{Introduction}

Restenosis, the main limitation of intracoronary stenting, can be defined as overreactive healing process of the endothelium against the mechanical insult. Neointimal hyperplasia inside the stent is the major contributor in restenosis ${ }^{[1]}$. Clinical and laboratory data show that local inflammatory process takes role in neointimal hyperplasia process ${ }^{[2]}$.

Apart from the local inflammation, systemic inflammatory markers are shown to increase after stent implantation. C-reactive protein (CRP), mainly synthesized in liver as an acute phase reactant, is a good systemic inflammatory marker. CRP is shown to peak at $48^{\text {th }}$ hour and down at $72^{\text {th }}$ hour to normal serum levels after successful coronary stenting in stable coronary artery disease (CAD) patients ${ }^{[3]}$. This increase in CRP levels is thought to be related with increased restenosis rates and adverse cardiac events ${ }^{[4]}$.

Understanding the pathophysiology of neointimal hyperplasia and restenosis has led to emergence of the antirestenotic strategies focusing on the cellular proliferation. Drug eluting stent (DES) technology is mainly based on the principal of eluting high concentrations of immunsuppresive or antitumor agents to the vessel wall. By the local effect of antiproliferative drug, neointimal proliferation and stent restenosis rates decreases. Today, restenosis rates are decreased by $80 \%$ after the introduction of DES, when compared with bare metal stents (BMS ${ }^{[5]}$. This decrease, achieved by DES, has been subject to investigation from the point of

Copy rights: (C) 2015 Nil, O. This is an Open access article distributed under the terms of Creative Commons Attribution 4.0 International License. 
inflammatory processes. There is no doubt of the effect of local anti inflammatory process on the vessel wall, but there is still no consensus about the possible systemic anti inflammatory effects of DES contributing its antirestenotic property.

In a trial by Kim JY et al. increase in CRP levels after DES impantation was significantly lower than the BMS group. Those were the first clinical data suggesting the possible beneficial effects of DES on systemic inflammation ${ }^{[6]}$. However, there were trials showing no significant CRP level difference between DES and BMS groups ${ }^{[7,8]}$. In addition to those trials done with first generation DES like sirolimus and paclitaxel, in 2014 a new trial with new generation DES, everolimus, was published. As a result of this recent trial, DES were shown to increase systemic inflammatory response more than the $\mathrm{BMS}^{[9]}$.

Our aim in this trial was to test the hypothesis that there would be less increase in post procedural CRP in DES group when compared to BMS group. We also analysed the relationship between post procedural CRP increase and CK-MB levels, a marker of procedure related myonecrosis. In addition, we searched for an answer for the possible relationship between post procedural CRP increase and cardiovascular prognosis in patients followed up for one year.

\section{Patients and Methods}

Patients who were admitted for elective coronary angiography for stable CAD andhad intracoronary stent placement for treatment, were evaluated for our study. Exclusion criteria were, patients with acute coronary syndrome, having percutenous coronary intervention or by-pass grefting in the last 2 weeks, with known infection or surgical intervention in the last 2 weeks, having a chronic inflammatory or autoimmune disease, having stent intervention to by-pass grefts, having stent intervention to restenosed stents, having both DES and BMS stent intervention in the same procedure and patients having treatment with glycoprotein IIb/IIIa inhibitor pre or post procedurely. Out of the evaluated 345 patients, 112 patients were included in our study. Written informed consent was obtained from each subject and the institutional ethics committee approved the study protocol.

Ten mililiters of venous blood for CRP and CK-MB was collected just before the intervention and at the $24^{\text {th }}$ hour after the procedure. The bloods were centrifuged (3000 g for 15 minutes) in 1 hour after collection and serums were kept in $-20^{\circ} \mathrm{C}$ in deep freezer. After the completetion of the patient inclusion, serum samples were sent to laboratory for CRP and CKMB assays. The CRP assays were analysed by Particle Enhanced Turbidimetric Process by using Cobas Integra reactive (Roche Cobas Integra 400 plus) with analytic senstivity of $0.1 \mathrm{mg} / \mathrm{L}$ (referance range of hsCRP:0-3 mg/L, test kits produced by Roche Diagnostics GmbH, D68298 Mannheim). CK-MB assays were analysed by kinetic spectrophotometric process after immunoinhibition by using Cobas Integra reactive (referance range: 0-6.25 $\mathrm{ng} / \mathrm{ml}$ ).

Biochemical work out and complete blood count were done after 12 hours of fasting, including the parameters of fasting blood glucose and lipid profile. Patients with LDL $>130$ $\mathrm{mg} / \mathrm{dl}$ or using anti hyperlipidemic medicine were accepted as hyperlipidemic. Patients with sistolic blood pressure $\geq 140$ $\mathrm{mmHg}$ and/or diastolic blood pressure $\geq 90 \mathrm{mmHg}$ for at least 2 measurements, or the patients with antihypertensive medications were accepted as hypertensive. Cigarette smoking was checked as positive if the patient had been smoking in the last 12 months. Patients with fasting blood glucose of $\geq 126 \mathrm{mg} / \mathrm{dl}$ for at least 2 times, or the patients who were already on insulin or oral antidiabetics therapy were accepted as diabetic.

At least 8 hours before the procedure, patients were loaded with $300 \mathrm{mg}$ clopidogrel, in addition to $300 \mathrm{mg}$ aspirin. In BMS group clopidogrel was used for one month and in DES group it was used for 12 months. Further usage of clopidogrel was dependent on the patient's own specialist's decision. During the procedure, choosing the stent type of either BMS or DES was done by the operator, according to the lesion type and patient's status. DES types used at the time of the study period were sirolimus, zotarilimus and paclitaxel stents.

Periprocedural infarct and necrosis was defined according to "Universal Definition of Myocardial Infarction" ${ }^{[10]}$. Morphological classification of the lesion types were done according to ACC/AHA lesion classification system ${ }^{[11]}$. Lesions with morphological types of $\mathrm{B} 2$ and $\mathrm{C}$ were accepted as complicated lesions. When there was an intervention for more than one lesion type including B2 or C type of lesion, the patient was accepted as acase with complicated morphology.

Patients were followed up at the $1^{\text {st }}, 6^{\text {th }}$ and $12^{\text {th }}$ month at the outpatient wards or by phone about their angina status, non fatal myocardial infarction (MI), any percutaneous interventions or death. If needed, further examination and treatment was done. Adverse cardiac events were defined as non fatal MI (including periprocedural infarcts), target vessel revascularization and death.

\section{Statistical analysis}

The Statistical Package for the Social Sciences 11.5 program (SPSS Inc., Chicago, IL, USA) was used for the statistical study. Shapiro Wilk test was used to test if the continous variables were normally distributed or not. Continous variables of normal distribution were given as mean \pm standard deviation, variables not distributed normally as CRP and CK-MB were shown as median ( $1^{\text {st }}$ quartile- $3^{\text {rd }}$ quartile). Variables between the groups were compared by Student t-test or Mann-Whitney U-test. Statistical significance of the change in laboratory values of basal and the $24^{\text {th }}$ hour within the group was checked by paired sample t-test or Wilcoxon signed-rank test. The difference between the basal and $24^{\text {th }}$ values of CRP and CK-MB was calculated and shown by delta $(\Delta)$ sign. Post procedural increase in CRP levels were also calculated as percent (\%) increase and assessed. For the $\triangle \mathrm{CRP}, \triangle \mathrm{CK}-\mathrm{MB}$ and \%CRP increase data, which were not normally distributed, median ( $1^{\text {st }}-3^{\text {rd }}$ quartile) values were used. To check the strength of relationship between continous variables Pearson's correlation coefficients were used. The chi-square test was used to assess differences between categorical variables. A p value less than 0.05 was considered significant.

\section{Results}

One hundred and twelve stable CAD patients ( 84 male, 28 female, mean age $62 \pm 11$ ), electively stented in our coronary angiography laboratory, were included in our study. Basal demographic and clinical characteristics of the patients in BMS and DES groups are shown in Table 1. Age, sex, hyperlipidemia, 
hypertension, smoking status, angiotensin converting enzyme inhibitor (ACE-I) or angiotensin receptor blocker (ARB), statin use were similar between groups. Diabetes mellitus (DM) frequency was significantly higher in patients of BMS group ( $\mathrm{p}=$ 0.004). Seventy of the patients had BMS, 42 of them had DES implantation intracoronarily. Table 2 shows the angiographic and procedural characteristics of the 2 groups. The frequency of complicated lesion morphology, multi vessel disease, multi vessel intervention, stent lengths (summation of the stent lengths were taken in multi stent interventions), stent diameters (mean diameter was taken in multi stent interventions), mean stent number used, mean lesion stenosis percent and maximal atmospheric pressure (maximum value was taken in multi stent interventions) were similar between BMS and DES groups. Patient frequency with multi stent intervention was also similar.

Table 1: Basal demographic and clinical characteristics of the patients in bare metal stent and drug eluting stent groups.

\begin{tabular}{|l|c|c|c|c|}
\hline & $\begin{array}{c}\text { BMS } \\
(\mathrm{n}=70)\end{array}$ & $\begin{array}{c}\text { DES } \\
(\mathrm{n}=42)\end{array}$ & $\begin{array}{c}\text { General } \\
(\mathrm{n}=112)\end{array}$ & $\mathrm{P}$ \\
\hline Age & $62 \pm 10$ & $61 \pm 11$ & $62 \pm 11$ & 0,653 \\
\hline Male & $49(70 \%)$ & $35(83 \%)$ & $84(75 \%)$ & 0,115 \\
\hline Diabetes mellitus & $32(46 \%)$ & $8(19 \%)$ & $40(36 \%)$ & 0,004 \\
\hline Hypertension & $53(76 \%)$ & $24(57 \%)$ & $77(69 \%)$ & 0,050 \\
\hline Hyperlipidemia & $49(70 \%)$ & $23(55 \%)$ & $72(64 \%)$ & 0,103 \\
\hline Smoking & $33(47 \%)$ & $21(50 \%)$ & $54(48 \%)$ & 0,770 \\
\hline ACE-Ior ARB & $53(76 \%)$ & $30(71 \%)$ & $83(74 \%)$ & 0,616 \\
\hline Statins & $65(93 \%)$ & $37(88 \%)$ & $102(91 \%)$ & 0,498 \\
\hline
\end{tabular}

Values are given as mean \pm standarddeviation or number (n) and $\%$. (ACE- $\mathrm{I}=$ angiotensin converting enzyme inhibitör, $\mathrm{ARB}=$ angiotensin receptor blocker, $\mathrm{BMS}=$ bare metal stent, $\mathrm{DES}=$ drug eluting stent)

Table 2: Angiographic and procedural characteristics of the patients in bare metal stent and drug eluting stent groups.

\begin{tabular}{|l|l|l|l|c|}
\hline & \multicolumn{1}{|c|}{$\begin{array}{c}\text { BMS } \\
(\mathrm{n}=70)\end{array}$} & $\begin{array}{c}\text { DES } \\
(\mathrm{n}=42)\end{array}$ & $\begin{array}{c}\text { General } \\
(\mathrm{n}=112)\end{array}$ & $\mathrm{p}$ \\
\hline Complicated lesion & $15(\% 21)$ & $14(\% 33)$ & $29(\% 26)$ & 0,164 \\
\hline $\begin{array}{l}\text { Multi vessel } \\
\text { disease }\end{array}$ & $38(\% 56)$ & $20(\% 50)$ & $58(\% 54)$ & 0,554 \\
\hline $\begin{array}{l}\text { Multi vessel } \\
\text { intervention }\end{array}$ & $22(\% 31)$ & $17(\% 40)$ & $39(\% 35)$ & 0,331 \\
\hline Stent length $(\mathrm{mm})^{*}$ & $25,6 \pm 20,69$ & $26,5 \pm 14,08$ & $25,9 \pm 18,43$ & 0,201 \\
\hline $\begin{array}{l}\text { Stent diameter } \\
(\mathrm{mm}) *\end{array}$ & $2,9 \pm 0,57$ & $2,9 \pm 0,33$ & $2,9 \pm 0,49$ & 0,946 \\
\hline $\begin{array}{l}\text { Mean number of } \\
\text { stents }\end{array}$ & $1,7 \pm 1,16$ & $1,4 \pm 0,70$ & $1,6 \pm 1,02$ & 0,247 \\
\hline $\begin{array}{l}\text { Mean percent of } \\
\text { stenosis \%* }\end{array}$ & $83,0 \pm 9,87$ & $80,6 \pm 9,77$ & $82,1 \pm 9,86$ & 0,238 \\
\hline $\begin{array}{l}\text { Max. atmospheric } \\
\text { pressure* }\end{array}$ & $13,1 \pm 1,94$ & $13,8 \pm 1,74$ & $13,4 \pm 1,88$ & 0,238 \\
\hline $\begin{array}{l}\text { Multi stent } \\
\text { intervention }\end{array}$ & $31(\% 44)$ & $13(\% 31)$ & $44(\% 39)$ & 0,162 \\
\hline
\end{tabular}

* Values are given as mean \pm standarddeviation.

$(\mathrm{BMS}=$ bare metal stent, DES $=$ drug eluting stent, Max.= maximum $)$
Preprocedural CRP levels were similar between the BMS and DES groups (4.4[2.1-10.7] mg/L, 3.6[1.1-6.6] mg/L, respectively, $p=0.120)$. When the $24^{\text {th }}$ hour values of CRP were checked, it was $8.6(4.1-16) \mathrm{mg} / \mathrm{L}$ for BMS and 6.4 (4.0-11.0) $\mathrm{mg} / \mathrm{L}$ for DES, $\mathrm{p}<0.001$, showing significant increase in both groups. Figure 1 shows the CRP level change between basal and $24^{\text {th }}$ hour in box-plot graphic. Increase in CRP levels from basal to $24^{\text {th }}$ hour were compared as $\Delta$ (delta) change and there were no significant difference between the groups $(\mathrm{p}=0.703)$. When the difference was calculated as percent $(\%)$ change, no significant difference between the goups was observed, either $(\mathrm{p}=0.374)$ (Table 3). To define the effects of demographic, angiographic and procedural factors contributing to $\triangle \mathrm{CRP}$ in all patients, correlation analysis was performed. Only with three of the procedural factors, which were multi vessel intervention, stent length and mean stent number, there was a trend for significance for the correlation with $\triangle \mathrm{CRP}(\mathrm{p}=0.058, \mathrm{p}=0.055$ and $\mathrm{p}=0.086$, respectively) (Table 4$)$. When we assessed the variables contributing to $\triangle \mathrm{CRP}$ in BMS and DES groups seperately, only the stent length variable was significantly correlated with $\triangle \mathrm{CRP}$ in DES group $(\mathrm{p}=0.016)$, whereas in BMS group stent length was not significantly related with $\triangle \mathrm{CRP}(\mathrm{p}=0.341)$ (Figure 2$)$.

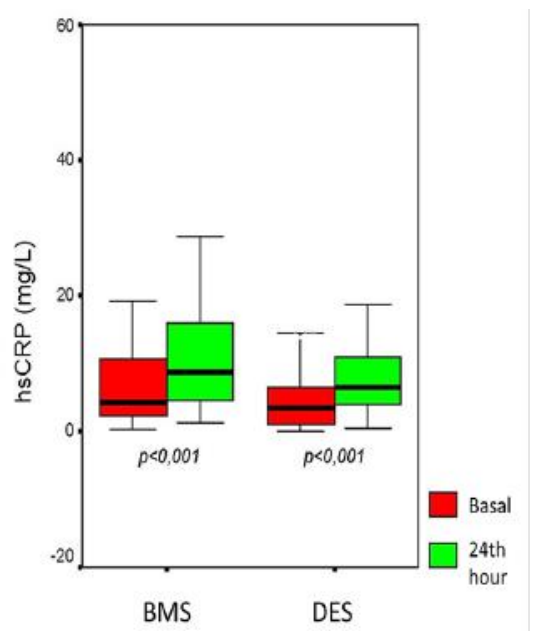

Figure 1: Basal and $24^{\text {th }}$ hour values of CRP in bare metal stent and drug eluting stent groups.(BMS = bare metal stent, DES $=$ drug eluting stent)

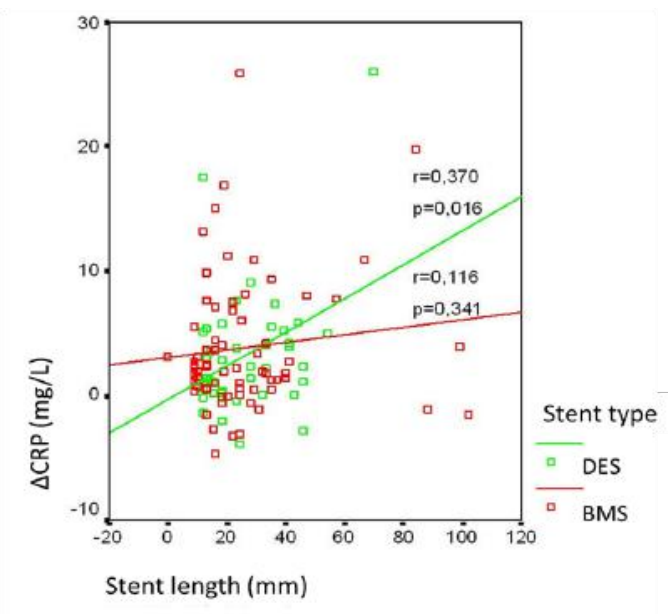

Figure 2: Correlation of stent length and $\triangle \mathrm{CRP}$ in bare metal stent and drug eluting stent groups. $(\mathrm{BMS}=$ bare metal stent, $\mathrm{DES}=$ drug eluting stent) 
Table 3: Comparison of the $\triangle \mathrm{CRP}$ and \% increase in CRP levels of the basal and the $24^{\text {th }}$ hour between the bare metal stent and drug eluting stent groups.

\begin{tabular}{|c|c|c|c|}
\hline & BMS $(\mathrm{n}=70)$ & DES $(\mathrm{n}=42)$ & $\mathrm{p}$ \\
\hline$\Delta \mathrm{CRP}(\mathrm{mg} / \mathrm{L})$ & $2,1(0,5-6,2)$ & $2,3(0,2-5,2)$ & 0,703 \\
\hline$\% \mathrm{CRP}$ increase & $50(14-152)$ & $74(6-245)$ & 0,374 \\
\hline
\end{tabular}

Values are given as median ( $1^{\text {st }}$ quartile- $3^{\text {rd }}$ quartile $)$

(BMS = bare metal stent, DES = drug eluting stent)

Table 4: Effects of the clinical, demographical, angiographic and procedural factors on levels of $\triangle \mathrm{CRP}$ in bare metal stent and drug eluting stent groups.

\begin{tabular}{|l|c|c|}
\hline & \multicolumn{2}{|c|}{ pvalues for $\Delta$ CRP } \\
\hline Age & BMS (n=70) & DES (n=42) \\
\hline Gender (female/male) & 0,446 & 0,885 \\
\hline Diabetes mellitus & 0,405 & 0,532 \\
\hline Hypertension & 0,693 & 0,224 \\
\hline Hyperlipidemia & 0,748 & 0,647 \\
\hline Smoking & 0,377 & 0,604 \\
\hline ACE-Ior ARB & 0,962 & 0,970 \\
\hline Statins & 0,130 & 0,436 \\
\hline Complicated lesion & 0,690 & 0,821 \\
\hline Multi vessel disease & 0,720 & 0,163 \\
\hline Multi vessel intervention & 0,161 & 0,626 \\
\hline Stent length (mm) & 0,101 & 0,311 \\
\hline Stent diameter (mm) & 0,341 & 0,016 \\
\hline Mean number of stents & 0,766 & 0,358 \\
\hline Max. atmospheric pressure & 0,285 & 0,102 \\
\hline Multi stent intervention & 0,302 & 0,172 \\
\hline
\end{tabular}

(ACE-I $=$ angiotensin converting enzyme inhibitör, $\mathrm{ARB}=$ angiotensin receptor blocker, $\mathrm{BMS}=$ bare metal stent, $\mathrm{DES}=$ drug eluting stent)

Preprocedural CK-MB levels were similar between the BMS and DES groups (3.2 [2.2-4.1] ng/ml, $2.6[2.0-3.7] \mathrm{ng} / \mathrm{ml}$, respectively, $p=0.160)$. When the $24^{\text {th }}$ hour values of CK-MB were checked, there were significant increase in both groups ( $p$ $<0.001$ for both groups). When CK-MB increase was calculated as $\triangle \mathrm{CK}-\mathrm{MB}$, there was no significant difference between the groups $(\mathrm{p}=0.897)$. Periprocedural myonecrosis (defined as postprocedure CK-MB as 1-3 times the upper normal limit) frequency was $17 \%$ in BMS, $12 \%$ in DES group $(p=0.269)$. Periprocedural MI frequency (defined as CK-MB increase more than 3 times the upper normal limit) was also similar between the BMS and DES groups ( $7 \%$ and $12 \%$, respectively, $p=0.219)$. In correlation analysis of 112 patients to assess the factors affecting $\triangle \mathrm{CK}-\mathrm{MB}$, only complicated lesion morphology was a contributor to CK-MB increase significantly $(\mathrm{p}=0.011)$. In detailed assessment of the stent groups seperately, complicated lesion morphology was seen to be a significant effector of $\triangle \mathrm{CK}-\mathrm{MB}$ only in BMS group $(\mathrm{p}=0.040)$, however this effect was not significant in DES group ( $\mathrm{p}=0.147)$.

We searched for the relationship between inflammation and myonecrosis. There was no significant correlation be- tween preprocedural CRP and $\triangle \mathrm{CK}-\mathrm{MB}$ levels $(\mathrm{r}=-0.004$ and $\mathrm{p}=0.965)$. There were also no correlation between $\triangle \mathrm{CRP}$ and $\Delta \mathrm{CK}-\mathrm{MB}$ levels $(\mathrm{r}=-0.007$ and $\mathrm{p}=0.941)$. When the data were assessed for $\triangle \mathrm{CRP}$ and $\triangle \mathrm{CK}-\mathrm{MB}$ correlation in BMS and DES groups seperately, no significant correlation was found, either $(\mathrm{r}=-0.090$ and $\mathrm{p}=0.459$ for BMS, $\mathrm{r}=0.158$ and $\mathrm{p}=0.318$ for DES).

During the follow up of the patients, 1 patient from the BMS group died in our coronary care unit at the $2^{\text {nd }}$ month of intervention because of end stage heart failure. At the end of first year, non-fatal MI (excluding periprocedural MI) and target vessel revascularization were in 7 patients in BMS and in 3 patients in DES group (10\% and 7\%, respectively, $\mathrm{p}=0.452)$. All adverse cardiac events, including non-fatal MI (including periprocedural MI), revascularization and death were $13(18 \%)$ in BMS and $9(22 \%)$ in DES group $(p=0.785)$. We assessed the possible effects of basal and $\triangle \mathrm{CRP}$ on adverse cardiac events for the 112 patients. Their effects on the event rates were not statistically significant $(\mathrm{p}=0.298$ and $\mathrm{p}=0.818$ for basal and $\Delta \mathrm{CRP})$.

\section{Discussion}

In our study searching for the relationship between stent type and systemic inflammatory response in 112 electively stented patients, we found similar amount of post procedure CRP increase in both BMS and DES groups. Also, periprocedural myonecrosis and adverse cardiac event rates were similar in both groups.

By the introduction of DES, the restenosis rates receded from $25 \%$ 's to $5 \%$ 's. Besides the known local anti inflammatory effects of DES, whether the systemic anti inflammation is also playing role at this benefit or not, has been subject to question. After the stent implantation, the plaque rupture and arterial wall injury trigger the inflammation and this can be assessed by the systemic inflammatory marker, $\mathrm{CRP}^{[12]}$. Increased CRP levels were shown to be related with increased restenosis rates. So, the researchers ask whether a systemic anti inflammatory property of DES has been contributing its anti restenotic property, or not. In a trial with 25 patients randomized to paclitaxel stent or BMS, restenosis was assessed by intravascular ulrasonography at the $6^{\text {th }}$ month. In both groups there were significant CRP and interleukin-6 (IL-6) increase at the $24^{\text {th }}$ hour compared to basal, but the difference between the stent groups was not significant. Also the rate of inflammation was not correlated with restenosis rates $^{[13]}$. Gaspardone et al. randomised the stable CAD patients to 4 groups; paclitaxel, sirolimus, dexamethasone eluting stents and BMS group. The groups were compared according to the CRP levels and the relation between the cardiovascular events. In all groups there were significant CRP increase, however, the amount of CRP increase was not significantly different between the groups. Restenosis and cardiovascular event rates were significantly lower in paclitaxel and sirolimus eluting stent groups. As a result of the trial, researchers concluded that the mechanism of the decrease in restenosis and adverse event rates in paclitaxel and sirolimus stent groups could not be related with the systemic inflammatory response ${ }^{[14]}$. In an other study assessing the systemic inflammatory response of DES, 300 patients were randomised to sirolimus eluting stents and BMS. $\triangle \mathrm{CRP}$ levels of the 2 stent groups were not different significantly ${ }^{[8]}$. In a trial including both stable CAD and acute coronary syndrome patients, 
34 patients had sirolimus stents and 29 patients had BMS. At the end of the trial CRP and IL-6 levels increased significantly in both stent groups, but the difference between the groups was not significant ${ }^{[7]}$. The trial done by Kim et al. concluded different results about the DES and systemic inflammation. They implanted BMS to 21 and DES to 46 stable CAD patients. Basal CRP levels were compared with the levels at $48^{\text {th }}$ and $72^{\text {th }}$ hour and the increase in CRP levels in DES group was found to be significantly lower. Those findings were the first data suggesting DES could have inhibitory effect on the acute postprocedure systemic inflammatory reaction ${ }^{[6]}$. Later, another trial with 257 BMS and 395 DES (sirolimus) implanted stable CAD patients were compared and DES group had significantly lower $\triangle \mathrm{CRP}$ levels. As a result, possible systemic anti inflammatory effects of DES, besides their local effects, were emphasized again ${ }^{[15]}$. In 2014, Liu et al. published a research about the same subject with the new generation stents ${ }^{[9]}$. Thirty six stable patients were randomised in 1:1 manner to everolimus type DES and BMS. Patients were assessed in the $12^{\text {th }}$ week of the procedure for their inflammatory levels by checking CRP, IL-6, tumor necrosis factor (TNF)- $\alpha$ and matrix metalloproteinase (MMP)-9 levels. In both stent groups CRP levels and lipid levels reduced at the 12 th week. In the DES group, different from the BMS group, levels of IL-6, TNF- $\alpha$ and MMP-9 levels increased. This trial is important, because it showed the continuing systemic inflammatory response in the DES group even at the $3^{\text {rd }}$ month. Also, those results showed that, IL-6, TNF- $\alpha$ and MMP-9 were more sensitive markers of inflammation, not being affected by statins as in the case of CRP.

In our study, we found that the systemic inflammation after DES was not less than the inflammation after BMS. These findings supported the hypothesis that DES do not reduce stent restenosis by systemicanti inflammatory effects and that had been supported by some trials before ${ }^{[7,8,13,14]}$. When the patient characteristics in our BMS group were investigated, it was noted that there were more diabetics in this group. This meant a more risky population were included when compared to the DES group, and this situation would have been expected to lead an increase in the inflammatory levels. However, the post procedure inflammation levels ended up to be the same with DES group. We may conclude that if the patient distribution were the same in both groups, DES group could have had a more pronounced inflammatory level increase than the BMS group. Some trials in the literature showed that, occasionally occuring vessel wall hypersensitivity reaction against the DES could make DES to have proinflammatory characteristics ${ }^{[16,17]}$. This hypersensitivity reaction could occur against the polymer, metal or the drug of DES. Eosinophyllic infiltration has been shown to be related with hypersensitivity reaction, however, there has been no exact data about its effects on CRP levels ${ }^{[17]}$. In recent years, new generation DES, like zotarilimus and everolimus has been introduced. They have a faster and shorter time of drug eluting technology, but also thought to stimulate the similar long term inflammatory response in body. This inflammation problem is hoped to be reduced by the newer bioabsorbable stent technology $^{[18]}$.

Our trial could not completely exclude the possibility of systemic anti inflammatory properties of DES. There was a possibility of the possible systemic anti inflammation by DES, shadowed by the basal patient characteristics and medical thera- pies used in the groups. We know the beneficial effects of statins on systemic inflammation and CRP levels and when we look at the patient characteristics we see nearly $90 \%$ of patients were on statins in both groups ${ }^{[19,20]}$. In a trial assessing the effects of pre procedural CRP levels on adverse cardiac events, the determinant effects of CRP dissappeared with statin use ${ }^{[21]}$. There is still no exact datain the literature about statin effects on CRP levels in postprocedure period, but, we suggest that high percentage of statin use in our trial could have masked the possible higher increase of CRP in BMS group, which had higher risk patients.

When we looked at the possible effects of angiographic and procedural factors, we saw that inflammation level was positively correlated with the stent length only in DES group. This could be explained by the increased inflammatory response secondary to increased foreign body reaction and endothelial damage. Why this effect could not be shown in the BMS group could be explained by the low patient number in the trial. However, it also could be explained by the hypothesis that increased metallic load in DES group could be a more potent trigger of the inflammation when compared to BMS group.

Minor increases in CK-MB levels, an indicator of myonecrosis, can be seen in $15-25 \%$ of the patients after succesful percutaneous interventions ${ }^{[22,23]}$. In our trial this rate was $15 \%$ and there was no significant difference between BMS and DES groups. Long lesions, occlusion of the side branches and distal emboli, which are especially the characteristics complicated lesions, lead to higher increase in CK-MB levels ${ }^{[24]}$. In our trial, parallel with those data, we found a significant relationship between complicated lesions and $\triangle \mathrm{CK}-\mathrm{MB}$. This relationship was shown in BMS group, could n't be shown in DES group, possibly because of the insufficient patient population.

We evaluated the patients of our trial in $1 \mathrm{st}, 6^{\text {th }}$ and $12^{\text {th }}$ months in our outpatient wards or got information by phoning about the adverse cardiac events. We did not make routine coronary angiographic assessment. Periprocedural MI in our trial was $10 \%$ and it was consistent with the data of other trials with 7-10\% periprocedural $\mathrm{MI}^{[7,21]}$. Adverse cardiac events frequency was not different between BMS and DES groups, similar to the published data ${ }^{[25]}$. Researchers investigated the relationship between adverse cardiac events and inflammation and myonecrosis levels postprocedurely ${ }^{[26,27]}$. In a trial, it was shown that early adverse cardiac events and restenosis rates were increased in the patient group with high preprocedural CRP levels ${ }^{[26}$. In another trial, patients with stable CAD and high preprocedural CRP levels had more increased levels of troponin after stenting ${ }^{[27]}$. In our results, there were no significant relationship of basal and $\triangle C R P$ levels with adverse cardiac events and $\triangle \mathrm{CK}-\mathrm{MB}$. This finding was not parallel with most of the trials showing the relationship between inflammation and adverse cardiac events in the literature. We could explain our results with similar findings of the trial by Chan et al. In this trial, they evaluated the effects of preprocedural CRP levels on the frequency of periprocedural MI and adverse cardiac events in 2 groups; the patients using statin and statin naive patients. In statin naive patients high basal CRP was an independent determinant of periprocedural MI and adverse cardiac events. However, there was no relationship between CRP levels and adverse cardiac events in statin using patients. As a result, they suggested that in statin users predictive value of CRP about the adverse cardiac events and periprocedural MI was blunted and diminished ${ }^{[21]}$. In assessment of our 
results, we could relate the non-significant relationship of the level of inflammation and adverse cardiac events with the high degree of statin use, in about $90 \%$ of the patients.

We believe our study revealed important data about the comparison of CRP levels between BMS and DES implanted patients and also searched for possible influence of many factors on levels of inflammation. In the literature, trials investigating the relation between the postprocedure CRP increase and adverse cardiac events and myonecrosis, are quite few. As a result of our trial, we concluded that there was no difference at the level of systemic inflammation between BMS and DES groups, in consistency with most of the published trials in this topic. DES show inhibitory effects on neointimal proliferation by local release of high concentrations of anti-inflammatory and anti-proliferative drugs. Beside the triggerred local inflammation, systemic inflammation also has detrimental effects on neointimal proliferation. In our study, we assessed the hypothesis of possible systemic anti-inflammatory effects of DES may play role in their anti-restenotic properties, besides their local effects and we concluded the result that DES did not have an inhibitory effect on systemic inflammation. DES lead to lower levels of restenosis than BMS at the same level of systemic inflammation. So, we suggest that this may be by local and prolonged resistance of DES to systemic inflammation when compared with BMS.

In our study including 112 stable CAD patients stented by BMS or DES, results revealed that DES had no suppressive effects on systemic inflammatory response. Recently, there has been published data about the prolonged and pronounced systemic inflammatory response triggered by DES, in contrary to their local anti-inflammatory properties ${ }^{[9]}$. When we have a consensus and exact data in this topic in the future, we may prescribe much intense anti-inflammatory regimens such as higher dose statins for the patients with DES. However, we need larger randomised trials to be able to clarify the exact mechanisms.

\section{Limitations}

The main limitation of our trial is that it was not randomised. At the time period of our trial, DES were not paid by the social security institution for every patient, so we weren't able to randomise the patients to each stent group. As a result, there were differences of patient characteristics between the stent groups, like more diabetics in BMS group. We tried to examine each factor, possibly effecting the results, seperately to be able to overcome this limitation.

Post procedural peak CRP levels are achieved at the $48^{\text {th }}$ hour, but we used the $24^{\text {th }}$ hour values ${ }^{[5]}$. Without difference in the $24^{\text {th }}$ hour values, no significant difference is accepted at the $48^{\text {th }}$ hour. So, most of the trials inthe literature also used the $24^{\text {th }}$ hour values, like we $\operatorname{did}^{[713,15]}$.

\section{Conflict of Interest}

Issues regarding the authorship or article: None declared.

\section{Financial Sources}

This trial was supported financially by "Clinical Vascular Biology Foundation, Turkey".

\section{References}

1. Virmani, R., Farb, A. Pathology of in-stent restenosis. (1999) Curr
Opin Lipidol 10(6): 499-506.

2. Welt, F.G., Tso, C., Edelman, E.R., Kjelsberg, M.A., et al. Leukocyte recruitment and expression of chemokines following different forms of vascular injury. (2003) Vasc Med 8(1): 1-7.

3. Versaci, F., Gaspardone, A. Prevention of restenosis after stenting: the emerging role of inflammation. (2004) Coron Artery Dis 15(6): $307-$ 311.

4. Gaspardone, A., Crea, F., Versaci, F., et al. Predictive value of C-reactive protein after successful coronary artery stenting in patients with stable angina. (1998) Am J Cardiol 82(4): 515-518.

5. Schwartz, R.S., Edelman, E.R., Carter, A., et al. Drug-eluting stents in preclinical studies: recommended evaluation from a consensus group. (2002) Circulation 106(14): 1867-1873.

6. Kim, J.Y., Ko, Y.G., Shim, C.Y., et al. Comparison of effects of drug-eluting stents versus bare metal stents on plasma C-reactive protein levels. (2005) Am J Cardiol 96(10): 1384-1388.

7. Gogo, P.B.Jr., Schneider, D.J., Watkins, M.W., et al. Systemic inflammation after drug-eluting stent placement. (2005) J Thromb Thrombolysis 19(2): 87-92.

8. Dibra, A., Ndrepepa, G., Mehilli, J., et al. Comparison of C-reactive protein levels before and after coronary stenting and restenosis among patients treated with sirolimus eluting versus bare metal stents. (2005) Am J Cardiol 95(10): 1238-1240.

9. Liu, J., Zhuo, X.Z., Liu, W., et al. Drug-eluting stent, but not bare metal stent, accentuates the systematic inflammatory response in patients. (2014) Cardiology 128(3): 259-265.

10. Thygesen, K., Alpert, J.S., White, H.D. Joint ESC/ACCF/AHA/ WHF Task Force for the Redefinition of Myocardial Infarction. Universal definition of myocardial infarction. (2007) Eur Heart J 28(20): 2525-2538

11. Ryan, T.J., Bauman, W.B., Kennedy, J.W., et al. Guidelines for percutaneous transluminal coronary angioplasty. A report of the American Heart Association/American College of Cardiology Task Force on Assessment of Diagnostic and Therapeutic Cardiovascular Procedures (Committee on Percutaneous Transluminal Coronary Angioplasty). (1993) Circulation 88(6): 2987-3007.

12. Azar, R.R., McKay, R.G., Kiernan, F.J., et al. Coronary angioplasty induces a systemic inflammatory response. (1997) Am J Cardiol 80(11): 1476-1478.

13. Klitkou, J., Jensen, L.O., Hansen, H.S., et al. High sensitive C-reactive protein and interleukin 6 are not related to neointimal hyperplasia in paclitaxel eluting stents or bare metal stents. An intravascular ultrasound study. (2007) Int J Cardiol 119(1): 114-116.

14. Gaspardone, A., Versaci, F., Tomai, F., et al. C-Reactive protein, clinical outcome, and restenosis rates after implantation of different drug-eluting stents. (2006) Am J Cardiol 97(9): 1311-1316.

15. Karha, J., Bavry, A.A., Rajagopal, V., et al. Relation of C-reactive protein level and long-term risk of death or myocardial infarction following percutaneous coronary intervention with a sirolimuseluting stent. (2006) Am J Cardiol 98(5): 616-618.

16. Nebeker, J.R., Virmani, R., Bennett, C.L., et al. Hypersensitivity cases associated with drug-eluting coronary stents: a review of available cases from the Research on Adverse Drug Events and Reports (RADAR) project. (20060 J Am Coll Cardiol 47(1): 175-181.

17. Kounis, N.G., Hahalis, G., Theoharides, T.C. Coronary stents, hypersensitivity reactions, and the Kounis syndrome. (2007) J Interv Cardiol 20(5): 314-323.

18. Gogas, B.D., McDaniel, M., Samady, H., et al. Noveldrug-eluting stents for coronary revascularization. (2014) Trends Cardiovasc Med 24(7): 305-313.

19. Libby, P., Ridker, P.M., Maseri, A. Inflammation and atherosclerosis. (2002) Circulation 105: 1135-1143.

20. Prasad, K. C-reactive protein (CRP)-lowering agents. (2006) Cardiovasc Drug Rev 24(1): 33-50.

21. Chan, A.W., Bhatt, D.L., Chew, D.P., et al. Relation of inflammation and benefit of statins after percutaneous coronary interventions. (2003) 
Circulation 107(13): 1750-1756.

22. Klein, L.W., Kramer, B.L., Howard, E., et al. Incidence and clinical significance of transient creatine kinase elevations and the diagnosis of non-Q wave myocardial infarction associated with coronary angioplasty. (1991) J Am Coll Cardiol 17(3): 621-626.

23. Ravkilde, J., Nissen, H., Mickley, H., et al. Cardiac troponin T and CK-MB mass release after visually successful percutaneous transluminal coronary angioplasty in stable angina pectoris. (1994) Am Heart J 127(1): 13-20.

24. Heyndrickx, G.R., Amano, J., Kenna, T., et al. Creatine kinase release not associated with myocardial necrosis after short periods of coronary artery occlusion in conscious baboons. (1985) J Am Coll Cardiol
6(6): 1299-1303.

25. Hill, R.A., Boland, A., Dickson, R., et al. Drug-eluting stents: a systematic review and economic evaluation. (2007) Health Technol Assess 11(46).

26. Buffon, A., Liuzzo, G., Biasucci, L.M., et al. Preprocedural serum levels of C-reactive protein predict early complications and late restenosis after coronary angioplasty. (1999) J Am Coll Cardiol 34(5): 15121521.

27. Goldberg, A., Gruberg, L., Roguin, A., et al. Preprocedural C-reactive protein levels predict myocardial necrosis after successful coronary stenting in patients with stable angina. (2006) Am Heart J 151(6): 1265 1270 . 